\title{
Renouvellement du carbone profond des sols cultivés : une estimation par compilation de données isotopiques
}

\author{
Jérôme Balesdent ${ }^{(1)}$, Isabelle Basile-Doelsch ${ }^{(1)}$, Joël Chadœuf ${ }^{(1,2)}$, Sophie Cornu ${ }^{(1)}$, \\ Zuzana Fekiacova ${ }^{(1)}$, Sébastien Fontaine ${ }^{(3)}$, Bertrand Guenet ${ }^{(4)}$, Christine Hatté ${ }^{(4)}$
}

\author{
(1) Aix-Marseille Univ., CNRS, Collège de France, IRD, INRA, CEREGE. Europôle de l'Arbois. BP80. FR-13545 Aix-en- \\ Provence cedex 04 (France). E-mail : jerome.balesdent@aix.inra.fr \\ (2) INRA. Centre de Recherche de PACA. Domaine St Paul - Site Agroparc. 228, Route de l'Aérodrome. CS 40509. FR- \\ 84914 AVIGNON Cedex 9 (France). \\ ${ }^{(3)}$ INRA UREP. 5, Chemin de Beaulieu. FR-63039 Clermont-Ferrand (France). \\ (4) Université Paris-Saclay. UMR 8212 CEA, CNRS, UVSQ. Laboratoire des Sciences du Climat et de l'Environnement. \\ Domaine du CNRS. FR-91190 Gif-sur-Yvette (France).
}

Reçu le 22 mars 2016, accepté le 6 septembre 2016.

Cet article est distribué suivant les termes et les conditions de la licence CC-BY (http://creativecommons.org/licenses/by/4.0/ deed.fr)

Description du sujet. L'article présente une estimation du renouvellement à moyen terme (dizaines d'années) du carbone profond des sols cultivés.

Objectifs. Le stockage de carbone sous forme organique dans les sols est un moyen de limiter l'augmentation du $\mathrm{CO}_{2}$ global et le réchauffement climatique. L'objectif est de proposer une première quantification du renouvellement des horizons profonds $(>30 \mathrm{~cm})$ qui contiennent environ la moitié du carbone organique des sols.

Méthode. Nous avons effectué une synthèse d'études de la dynamique du carbone de 41 profils où le renouvellement du carbone est quantifié grâce au traçage isotopique par les abondances naturelles en carbone-13.

Résultats. Le renouvellement du carbone profond est en moyenne quatre fois plus lent qu'en surface, mais le flux de renouvellement n'est pas négligeable. La distribution moyenne du flux annuel d'apport de carbone aux matières organiques est de $81 \%$ dans l'horizon $0-30 \mathrm{~cm}$ et $19 \%$ dans la couche $30-100 \mathrm{~cm}$, avec une erreur standard de $\pm 4 \%$. À plus long terme (20 ans), le sous-sol (30-100 cm) contient en moyenne $23 \%$ du carbone récent du sol.

Conclusions. Les matières organiques profondes ne doivent pas être négligées dans les bilans de $\mathrm{C}$ et $\mathrm{N}$ en réponse à différentes pratiques agricoles, et nous proposons une approximation simple pour ces bilans. Dans le futur, les estimations des évolutions temporelles du stock de carbone profond des sols et de leurs facteurs de variation devront s'appuyer sur les essais agronomiques de longue durée ou la modélisation calée sur des sites fortement instrumentés, et être accompagnées de descriptions pédologiques détaillées.

Mots-clés. Matière organique du sol, expérimentation au champ, sous-sol, isotope naturel, plante en C4.

\section{Turnover of deep organic carbon in cultivated soils: an estimate from a review of isotope data}

Description of the subject. This study is an estimate of medium-term renewal (decades) of deep carbon in cultivated soils. Objectives. Sequestration of organic carbon in soils is a way to mitigate the global rise in $\mathrm{CO}_{2}$ and warming. Deep horizons $(>30 \mathrm{~cm}$ ) contain about half of the soil organic carbon but, in agriculture, deep organic carbon and nitrogen are often neglected when the balance of these elements is calculated. Our goal in this study was to propose a quantification of deep C turnover.

Method. We collected data from 41 profiles, where $\mathrm{C}$ turnover was studied using the natural ${ }^{13} \mathrm{C}$ labeling technique. The studied cropping systems were basically tropical and temperate $\mathrm{C} 4$ plant monocultures.

Results. Deep carbon $(30-100 \mathrm{~cm})$ turnover was on average four times slower than in topsoil $(0-30 \mathrm{~cm})$, but renewal was significant. The average depth-distribution of the carbon flux to organic matter build-up was $81 \%$ in the layer at the $0-30 \mathrm{~cm}$ level and $19 \%$ in the layer at $30-100 \mathrm{~cm}$, with a standard error of $\pm 4 \%$. Over the longer term (20 years), subsoil at $30-100 \mathrm{~cm}$ contained on average $23 \%$ of the recent soil carbon accumulated in the first meter.

Conclusions. Deep organic matter should not be overlooked when considering the balance of $\mathrm{C}$ and $\mathrm{N}$ and we therefore propose a simple method for an initial first assessment. In the future, further estimates of the temporal evolution of the deep 
carbon stock and its factors of variation should be based on long-term agronomic experiments or modeling parameterized on heavily instrumented sites, and should be accompanied by detailed pedological descriptions.

Keywords. Soil organic matter, field experimentation, subsoil, natural isotopes, C4 plants.

\section{INTRODUCTION}

La question de la dynamique du carbone organique profond des sols est posée actuellement par la communauté scientifique qui évalue le cycle planétaire du carbone en lien avec l'évolution du climat. La taille du réservoir de carbone des sols de la planète est évaluée entre 1500 Gt C et 2400 Gt C selon les auteurs et les profondeurs prises en compte (Hiederer et al., 2011 ; Ciais et al., 2013), hors zone de permafrost (Koven et al., 2011), ce qui en fait un puits ou une source potentielle énorme par rapport à l'augmentation du $\mathrm{CO}_{2}$ atmosphérique $(+4,4 \mathrm{Gt} \mathrm{C}$ en 2015). Or, à l'échelle planétaire comme à l'échelle de la France, la moitié du carbone est située en dessous de $30 \mathrm{~cm}$ de la surface du sol (Jobbagy et al., 2000 ; Hiederer et al., 2011 ; Mulder et al., 2016). Même si les teneurs en carbone sont plus basses qu'en surface, la masse volumique apparente plus élevée et l'épaisseur parfois importante du sol font que les stocks 0-30 et 30-100 cm sont du même ordre de grandeur.

L'incertitude sur la dynamique du carbone des sols est souvent invoquée pour expliquer la relative mauvaise représentation du carbone des écosystèmes dans les modèles globaux, qu'il s'agisse de représenter les flux nets, le turnover ou le stock (Ahlström et al., 2012 ; Carvalhais et al., 2014). En effet, la dynamique du carbone superficiel est relativement bien quantifiée, en particulier grâce aux expérimentations de longue durée, dont les plus anciennes ont été initiées à la fin du $19^{\mathrm{e}}$ siècle, alors qu'il reste des interrogations majeures sur l'estimation du renouvellement du carbone des sols situé en dessous des 30 premiers centimètres et les processus en jeu (Harper et al., 2013), ce qui en fait une source d'incertitude majeure pour les prévisions sur l'évolution du cycle global du carbone et du climat.

Plusieurs auteurs ont démontré que les mécanismes présidant à la préservation ou à la biodégradation peuvent s'exprimer avec des intensités différentes en surface et en profondeur (Fontaine et al., 2007 ; Salomé et al., 2010). En agriculture, la partie profonde des sols est particulièrement surveillée et gérée pour ses propriétés physiques, mais en général peu considérée en tant que réserve de matière organique, ou comme source ou puits d'éléments nutritifs. Les modèles opérationnels du bilan organique utilisés en agriculture, comme RothC (Coleman et al., 1995), ou AMG (Andriulo et al., 1999 ; Bouthier et al., 2014) ne considèrent d'ailleurs que la couche superficielle (0 - $30 \mathrm{~cm}$ dans l'Union européenne), alors que la distribution du carbone en fonction de la profondeur est un continuum, à la seule exception de l'horizon Ap où la distribution du $\mathrm{C}$ peut être homogène lorsqu'il $\mathrm{y} a$, ou qu'il y a eu dans le passé, labour par retournement.

Le renouvellement du carbone profond est lent, comme en atteste la datation au ${ }^{14} \mathrm{C}$ : l'âge apparent ${ }^{14} \mathrm{C}$, proche de l'âge moyen du carbone, atteint couramment 2500 ans à $1 \mathrm{~m}$ de profondeur (Mathieu et al., 2015). Ceci rend très difficile l'étude de sa dynamique par le suivi temporel des stocks. Les flux bruts sont également difficilement mesurables. Concernant les flux entrants, il s'agit essentiellement de mortalité racinaire, de rhizodéposition et de flux verticaux de matière organique soluble, qui sont très difficiles à estimer : le renouvellement racinaire peut être quantifié en longueur ou volume de racines, mais plus difficilement en masse et le caractère transitoire des rhizodépôts et exsudats les rend insaisissables sans traçage isotopique (Balesdent et al., 2011).

S'agissant du flux sortant du réservoir organique, principalement la minéralisation sous forme de $\mathrm{CO}_{2}$, sa quantification in situ est rendue très délicate voire impossible car l'efflux de $\mathrm{CO}_{2}$ comprend également la respiration autotrophe racinaire, qui est neutre pour le bilan organique du sol, mais souvent supérieure à la respiration hétérotrophe des décomposeurs de matière organique (Hanson et al., 2000). Enfin, il est quasiment impossible d'accéder à la minéralisation du $\mathrm{C}$ profond sans induire une perturbation majeure des processus par la mise en place de capteurs, l'exclusion des racines ou le prélèvement de sol dans les horizons profonds.

Une méthode de choix pour l'investigation de la dynamique du carbone est le traçage isotopique naturel: les variations d'abondance naturelle en ${ }^{13} \mathrm{C}$, un isotope stable du carbone, peuvent être exploitées dans le cas de cultures de plantes à cycle photosynthétique $\mathrm{C} 4$ établies sur des sols ayant porté auparavant des couverts de végétation C3 (Cerri et al., 1985). Les plantes C4 les plus couramment cultivées sont le maïs, le sorgho, le mil, la canne à sucre et des plantes à vocation énergétiques comme Miscanthus et Panicum virgatum (switchgrass). La différence de rapport ${ }^{13} \mathrm{C} /{ }^{12} \mathrm{C}$ entre plantes $\mathrm{C} 3$ et $\mathrm{C} 4$ est importante, si bien que, après $t$ années de changement de végétation, la proportion de carbone organique provenant de la nouvelle végétation peut être déterminée à partir du rapport ${ }^{13} \mathrm{C} /{ }^{12} \mathrm{C}$ organique du sol. L'évolution de ce rapport en fonction du temps $t$ est une mesure directe du renouvellement du carbone du sol.

L'objectif de cette étude est de quantifier la répartition verticale des flux entrants, la variation des vitesses de renouvellement en fonction de la profondeur 
et, finalement, la contribution du carbone qui se trouve en profondeur, au pool de carbone "actif » des sols. Nous avons effectué, pour ce faire, la méta-analyse des données de 41 sites extraites de 25 études qui ont estimé le renouvellement du carbone le long de profils de sols cultivés au moyen du traçage isotopique par les abondances naturelles en ${ }^{13} \mathrm{C}$.

\section{MATÉRIEL ET MÉTHODES}

\subsection{Sites et études sélectionnées}

La technique du traçage par les abondances naturelles en carbone ${ }^{13} \mathrm{C}$ fait appel à deux parcelles qui ont porté la même végétation avant la mise en place des monocultures : la parcelle sous monoculture $\mathrm{C} 4$ et une parcelle de référence maintenue sous la végétation initiale ou mise en même temps sous végétation C3.

Les études ont été sélectionnées selon plusieurs critères : la durée de la monoculture doit être connue précisément, le sol doit avoir été échantillonné jusqu'à au moins $70 \mathrm{~cm}$ ou à la roche mère, les concentrations en carbone de la parcelle C4 doivent avoir été mesurées. Nous avons compilé les données publiées de 41 situations, tirées de 25 références (Annexe 1), où a varié la durée des monocultures de plantes $\mathrm{C} 4$ établies sur sols ayant porté au préalable des végétations de type C3. La base de données comporte quatre grands types de systèmes de culture (Tableau 1). Les principaux grands types de sols (IUSS Working Group WRB, 2014) représentés sont : cambisols (non andic inceptisols) $(\mathrm{n}=9)$, ferralsols et acrisols (oxisols et ultisols) $(\mathrm{n}=9)$, andosols $(\mathrm{n}=8)$, luvisols (alfisols) $(\mathrm{n}=7)$, chernozems (mollisols) $(\mathrm{n}=3)$, gleysols $(\mathrm{n}=$ $3)$, podzols $(n=2)$.

Tableau 1. Grands types de systèmes de culture exploités, nombre de sites, durée des observations - Studied cropping systems, number of sites and duration of observation.

\begin{tabular}{llc}
\hline & Nombre de sites & Durée (ans) \\
\hline $\begin{array}{l}\text { Monoculture de } \\
\text { maïs-grain en milieu } \\
\text { tempéré }\left(\leq 13^{\circ} \mathrm{C}\right)\end{array}$ & 16 & 8 à 37 \\
$\begin{array}{l}\text { Miscanthus en milieu } \\
\text { tempéré }\end{array}$ & 8 & 2 à 16 \\
$\begin{array}{l}\text { Monoculture de canne } \\
\text { à sucre en milieu } \\
\text { tropical }\left(\geq 17^{\circ} \mathrm{C}\right)\end{array}$ & 14 & 12 à 186 \\
$\begin{array}{l}\text { Maïs et rotations avec } \\
\text { maïs-grain en milieu } \\
\text { tropical }\left(\geq 17^{\circ} \mathrm{C}\right)\end{array}$ & 3 & 20 à 76 \\
\hline
\end{tabular}

\subsection{Calculs}

Le traçage isotopique permet, pour tout échantillon, de calculer la proportion $f$ de carbone provenant de la plante cultivée depuis le début de la monoculture, c'està-dire de carbone plus jeune que $t$ années. Le reste (1- $f$ ) est la proportion de carbone provenant des matières organiques présentes dans le sol avant le changement de végétation, qui n'a pas été minéralisée. Les stocks de carbone total, la proportion de carbone nouveau, provenant de la culture et les stocks de carbone nouveau ont été calculés de la manière suivante. Pour chaque couche $\left(z_{1}, z_{2}\right), C$ est le stock de carbone de la couche $\left(\mathrm{kg} \mathrm{C} \cdot \mathrm{m}^{-2}\right), f$ est la proportion de carbone nouveau provenant de la culture et $C_{\text {nouv }}$ est le stock de carbone nouveau $\left(\mathrm{kg} \mathrm{C} \cdot \mathrm{m}^{-2}\right)$. Les valeurs de $C, f$ et $C_{\text {nouv }}$ sont soit fournies par les auteurs, soit calculées à partir des concentrations en carbone $[C]\left(\mathrm{g} \mathrm{C} \mathrm{kg}^{-1}\right)$ et de la masse volumique apparente $\rho$, comme suit :

$$
C=[C] \cdot \rho \cdot\left(z_{2}-z_{1}\right)
$$

où p est fournie par les auteurs, ou calculée (Alexander, 1980). Les éléments grossiers sont négligés. Les variables $f$ et $C_{\text {nouv }}$ sont calculées d'après l'équation de Balesdent et al. (1996) qui relie la composition isotopique du carbone organique à la proportion de carbone provenant de la nouvelle végétation de type C4 :

$$
\begin{aligned}
& f=\left(\delta s o l-\delta s o l_{\text {ref }}\right) / \Delta v e g \\
& C_{\text {nouv }}=f \cdot C
\end{aligned}
$$

(Équation 2)

où $\delta$ sol et $\delta$ sol $_{\text {ref }}$ sont les rapports ${ }^{13} \mathrm{C} /{ }^{12} \mathrm{C} \mathrm{du}$ carbone de la couche considérée dans le sol étudié et le sol de référence, respectivement, et $\Delta v e g$ est la différence des rapports ${ }^{13} \mathrm{C} /{ }^{12} \mathrm{C}$ entre la végétation $\mathrm{C} 4$ et la végétation $\mathrm{C} 3$ de référence. En raison des enrichissements naturels en ${ }^{13} \mathrm{C}$ avec la profondeur, la valeur de $\delta s o l_{\text {ref }}$ est obtenue pour chaque couche à la même profondeur. L'équation 2 prend en compte les sources d'enrichissement isotopique autres que le changement de végétation, soit les variations isotopiques des végétations du passé et celles résultant de la biodégradation, et donne une estimation sans biais de $f$ si ces deux effets sont identiques dans les deux profils (Balesdent et al., 1996).

Afin de comparer les sites entre eux, nous avons intégré selon $z$ les stocks cumulés $C(0, z), C_{\text {nouv }}(0, z)$, puis calculé $C$ et $f$ par intervalle de $10 \mathrm{~cm}$ jusqu'à $100 \mathrm{~cm}$ par simple interpolation linéaire des stocks cumulés. Dans quelques cas de couches manquantes, les concentrations en carbone ont été interpolées à partir des couches supérieure et inférieure. Dans le cas de sites décrits jusqu'à une profondeur inférieure 
à $100 \mathrm{~cm}$ (le plus souvent $80 \mathrm{~cm}$ ), nous avons procédé à une extrapolation pour rapporter le stock de carbone des couches observées au stock de la profondeur de référence $0-100 \mathrm{~cm}$ : nous avons extrapolé $\mathrm{C}(0$, $100 \mathrm{~cm}$ ) à partir de la profondeur maximale décrite $z_{\max }$ selon la régression paramétrée pour l'ensemble des sites :

$$
\mathrm{C}(0,100 \mathrm{~cm})=\mathrm{a} * \mathrm{C}\left(0, z_{\max }\right)+\mathrm{b} .
$$

\subsection{Analyses statistiques}

Les régressions sont effectuées en utilisant le module d'ajustement par modèle linéaire $l m$ du logiciel R.

\section{RÉSULTATS}

\subsection{Vitesses de renouvellement et âge du carbone en surface et en profondeur}

La figure 1 présente la proportion de carbone nouveau $f$ en fonction du temps $t$, c'est-à-dire le renouvellement du carbone, dans les deux couches 0-30 et $30-100 \mathrm{~cm}$. Elle montre pour la couche supérieure quelques grandes tendances connues de la dynamique du carbone des sols. Le renouvellement du carbone est plus rapide dans les premières années, en raison de la présence de fractions rapidement décomposables et renouvelables, puis est de plus en plus lent, sans atteindre $100 \%$ même après plusieurs siècles, en raison de la présence de carbone résistant ou protégé de la minéralisation à très long terme. L'âge médian du carbone (temps correspondant au renouvellement de $50 \%$ ) est de l'ordre de 100 ans pour la couche $0-30 \mathrm{~cm}$. Le renouvellement du carbone est bien sûr très variable entre les sites. La variabilité, non discutée ici, est d'abord liée à trois facteurs : les phases minérales présentes, avec les minéraux comme les argiles ou les allophanes ralentissant le renouvellement; le climat, avec un renouvellement plus rapide en conditions tropicales; la différence de dynamique du carbone entre la monoculture étudiée et le système ou l'usage qui la précédait, qu'il s'agisse du changement des flux de restitution de carbone au sol ou des vitesses de minéralisation. Le renouvellement en profondeur est beaucoup plus lent. Sur chaque site, la proportion de carbone nouveau décroît progressivement avec la profondeur, jusqu'à une valeur de quelques $\%$ à $100 \mathrm{~cm}$. Nous avons exprimé le renouvellement de la couche $30-100 \mathrm{~cm}$ des mêmes sites (Figure 1). En moyenne, la proportion de carbone nouveau dans la couche $30-100 \mathrm{~cm}$ est trois fois plus faible $(2,9$ avec un écart-type de 1,9$)$ que dans la couche $0-30 \mathrm{~cm}$. Des régressions exponentielles de type $f=\mathrm{a}^{*}\left(1-\mathrm{e}^{-\mathrm{k} . t}\right)$ ont été effectuées sur l'ensemble

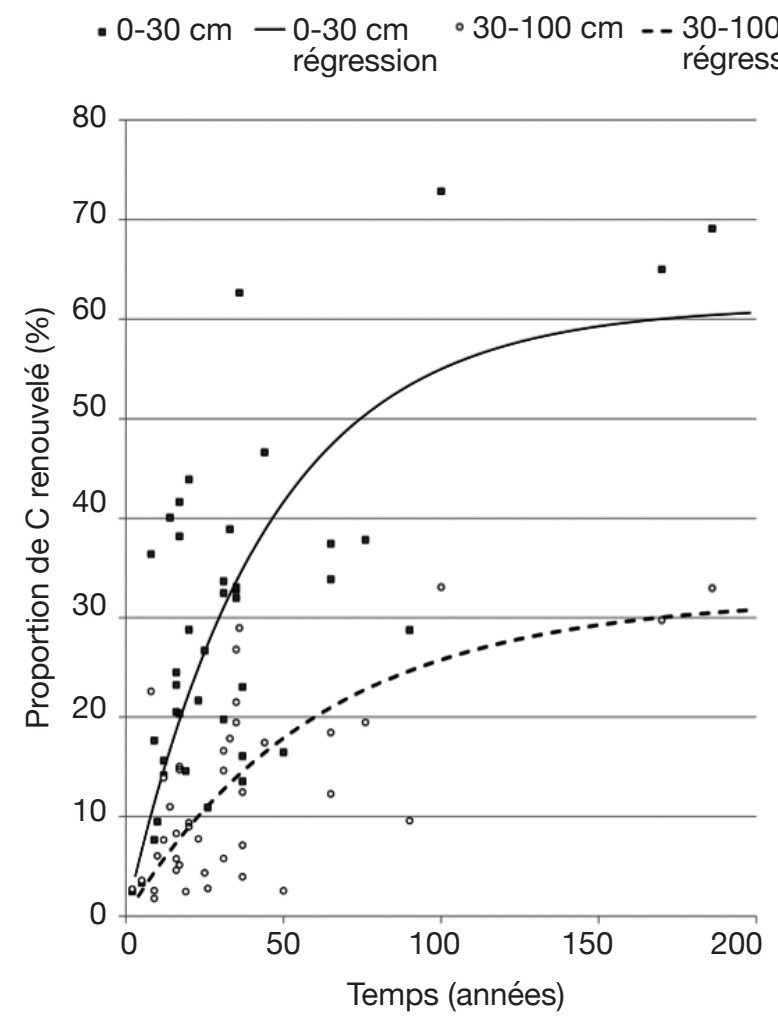

Figure 1. Renouvellement du carbone du sol pour deux couches (0-30 et $30-100 \mathrm{~cm}$ de profondeur) de 41 sites de sols cultivés. La proportion de carbone nouveau, provenant de la culture, est exprimée en fonction de la durée de la culture. Elle a été mesurée par les abondances naturelles en ${ }^{13} \mathrm{C}$ de monocultures de plantes $\mathrm{C} 4$. Les équations de régression sont : $f(0-30)=0,61 .\left(1-\mathrm{e}^{-0,023 . t}\right) ; \mathrm{r}^{2}=0,45 ; \mathrm{f}(30$ $-100)=0,32 .\left(1-\exp ^{-0,016 . t}\right) ; r^{2}=0,44-$ Carbon turnover for two depth-layers (topsoil 0-30 cm; subsoil $30-100 \mathrm{~cm}$ ) over 41 cultivated sites. Renewed carbon fraction is presented versus the culture duration. It has been determined by the natural ${ }^{13} \mathrm{C}$ labelling technique in $\mathrm{C} 4$ plant monocultures. Regression equations: $\mathrm{f}(0-30)=0.61 *\left(1-e^{-0.023 . t}\right) ; r^{2}=$ 0,$45 ; f(30-100)=0,32 .\left(1-\exp ^{-0,016 . t}\right) ; r^{2}=0,44$.

des points (Figure 1). Elles peuvent être interprétées comme décrivant grossièrement le renouvellement de la manière suivante : une proportion $(a)$ du carbone est sujette à renouvellement, le reste (1- $a$ ) étant stable à cette échelle de temps (200 ans); le taux moyen de renouvellement annuel de la partie renouvelée est $k\left(\mathrm{an}^{-1}\right)$; il s'agit de renouvellement par les nouveaux apports par la végétation qui compensent des départs par minéralisation et migration. A partir de ces régressions, on estime que le temps nécessaire pour renouveler $20 \%$ du carbone de l'horizon est en moyenne de l'ordre de trois à quatre fois plus long (environ 60 ans) pour $30-100 \mathrm{~cm}$ que pour $0-30 \mathrm{~cm}$ (environ 17 ans). Plusieurs hypothèses ont été avancées pour expliquer le renouvellement plus lent en profondeur (Rumpel et al., 2011 ; Guenet et al., 
2013). Le carbone stable à l'échelle du millénaire est en proportion relative plus importante en profondeur qu'en surface et, à la durée de vie du carbone in situ, il faut ajouter le temps nécessaire au transport de carbone depuis la surface vers la profondeur, par pédoturbation, bioturbation ou lixiviation (Elzein et al., 1995 ; Guenet et al., 2013). Enfin, la biodégradation peut être plus lente qu'en surface, en raison de plus fortes interactions organo-minérales (Salomé et al., 2010) ou de moindre capacité des micro-organismes à dégrader les matières organiques car ces derniers reçoivent moins de ressources énergétiques de substrats frais (Fontaine et al., 2007). Ces hypothèses doivent être considérées comme en interaction entre elles, aucune n'excluant les autres.

\subsection{Distribution verticale du carbone organique total}

La mesure des concentrations en carbone et de la densité apparente permet de calculer la répartition verticale du stock de carbone (Figure 2). Les concentrations volumiques en carbone sont en moyenne trois fois plus faibles à $1 \mathrm{~m}$ que dans l'horizon $\mathrm{A}$. Les concentrations sont très semblables au sein de la couche $0-30 \mathrm{~cm}$ car la plupart des sites ont été labourés soit régulièrement, soit occasionnellement, ce qui homogénéise les teneurs en carbone jusqu'à la profondeur de labour (20 à 30, parfois $40 \mathrm{~cm}$ ). Les horizons profonds (30$100 \mathrm{~cm}$ ) contiennent en moyenne $48 \%$ du carbone organique total du premier mètre, chiffre très proche des estimations à l'échelle de la France métropolitaine

Carbone organique total (\% du stock 0-100 cm)

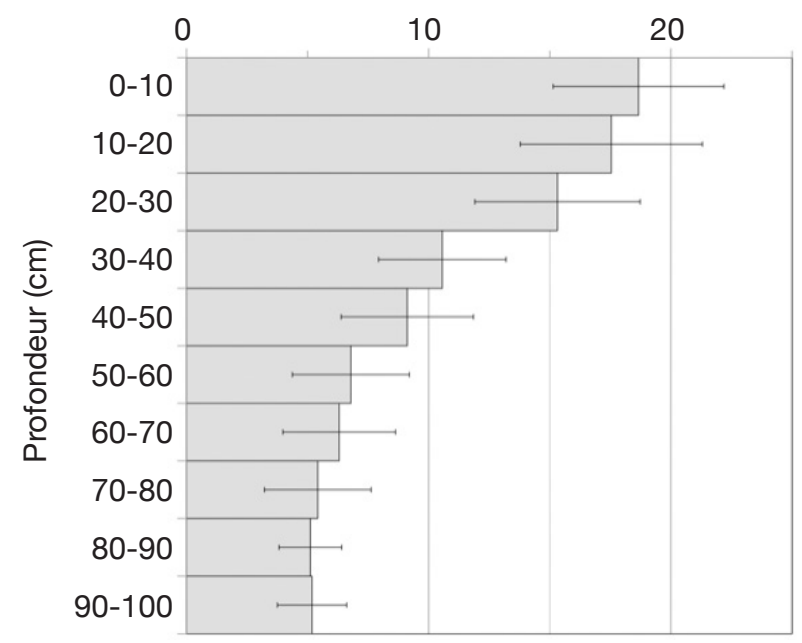

Figure 2. Répartition verticale du stock de carbone organique des sols étudiés (en \% du stock total 0-100 cm), moyennes et écarts-types - Depth distribution of total organic carbon stock in the studied soils (in \% of the stock $0-100 \mathrm{~cm}$ ), mean and standard deviation.
(Mulder et al., 2016) ou de la planète (Jobbagy et al., 2000). Au sein de l'ensemble des sols cultivés étudiés, cette proportion est assez variable $(1 \sigma=10 \%)$ et il est attendu que l'accumulation de carbone dans les horizons les plus profonds soit plus dépendante du type pédologique que de la culture elle-même. En effet, les travaux de Mathieu et al. (2015) ont montré au niveau de la planète que le degré de stabilisation (l'âge) du carbone profond est plus dépendant du type de sol que du climat ou de l'usage des terres.

\subsection{Distribution verticale du carbone provenant de la culture}

Nous avons estimé pour chaque couche le stock de carbone récent, à partir des abondances en ${ }^{13} \mathrm{C}$ et de la concentration volumique en carbone, et calculé pour chaque profil la répartition verticale de ce carbone récent, en \% du stock de carbone récent de l'ensemble du profil (méthode en Annexe 1). Ces données sont regroupées par culture sur la figure 3. Le rapport du carbone nouveau dans la couche $30-100 \mathrm{~cm}$ sur le carbone nouveau de l'ensemble $0-100 \mathrm{~cm}$ est en moyenne de 0,24 pour le maïs, 0,22 pour Miscanthus et 0,36 pour la canne à sucre.

La proportion du carbone nouveau localisé en profondeur dépend de plusieurs variables, notamment le temps d'observation et l'usage des sols. Ainsi, elle est plus importante pour les temps longs et dans les systèmes sous canne à sucre que sous cultures de maïs

\section{Carbone provenant de la plante cultivée} (\% du total $0-100 \mathrm{~cm}$ )

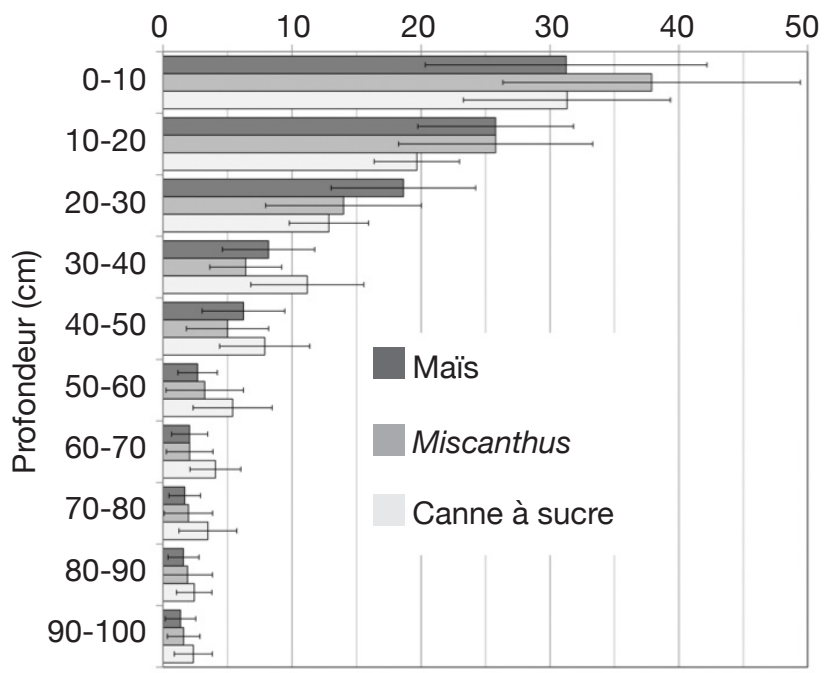

Figure 3. Répartition verticale du carbone provenant de la plante cultivée dans trois systèmes de cultures, moyennes et écarts-types - Depth distribution of crop-derived carbon in three cropping systems, mean and standard deviation. 
ou de Miscanthus ( $p<0,001)$, ce qui est visible sur la figure 3. La variation peut être expliquée par d'autres facteurs que la distribution des restitutions de carbone ou les enracinements. En effet, le jeu de données présente une covariance statistique entre la nature de la culture et le climat bien sûr, mais aussi entre la nature et la durée de la monoculture. La durée médiane des études est de 11, 26 et 47 ans pour Miscanthus, maïs et canne à sucre, respectivement. Plusieurs facteurs peuvent ainsi expliquer l'accumulation supérieure que nous observons en profondeur sous canne à sucre. La durée d'observation étant plus importante, l'accumulation relative en profondeur est supérieure. D'autre part, le brûlage des feuilles de canne a pu être largement pratiqué, notamment dans le passé, réduisant d'autant les apports en surface.

Pour analyser plus finement la dépendance du temps dans les systèmes sous Miscanthus et maïs, nous avons choisi comme indicateur unique d'incorporation le rapport $R$ du stock de carbone nouveau de la couche profonde sur le stock de carbone nouveau de l'ensemble du profil :

$$
R=\mathrm{C}_{\text {nouv }}(30-100 \mathrm{~cm}) / \mathrm{C}_{\text {nouv }}(0-100 \mathrm{~cm}),
$$

et nous avons analysé sa variation par un modèle de régression linéaire et une analyse de variance. L'équation de régression linéaire en fonction du temps et du système de culture est la suivante :

$$
R=a+b \cdot \text { temps }+ \text { résidu }
$$

avec $a$, dépendant du type de culture, $b$ commun.

L'estimation des paramètres est donnée au tableau 2. Les trois systèmes comparés sont très semblables. In fine, la régression linéaire simple, unique pour les systèmes en maïs et Miscanthus, en donne une bonne description :

Tableau 2. Régression par modèle linéaire du rapport $R$ du stock de carbone nouveau de la couche $30-100 \mathrm{~cm}$ au stock de carbone nouveau de la couche 0-100 cm des sols en fonction du temps et du système de culture. Le modèle est du type $R=a$ (système de culture $)+b$.temps + résidus - Linear regression of the ratio $\mathrm{R}$ of new carbon in the $0-30 \mathrm{~cm}$ layer to the new carbon in the $0-100 \mathrm{~cm}$ layer, as a function of time and cropping system. The model is $\mathrm{R}=\mathrm{a}$ (cropping system) + b.time + residuals.

\begin{tabular}{lll}
\hline Paramètres de la régression & Valeur du paramètre & Erreur standard \\
\hline$a$ (maïs, tempéré) & 0,189 & 0,051 \\
$a$ (maïs, tropical) & 0,216 & 0,088 \\
$a$ (Miscanthus) & 0,197 & 0,040 \\
\hline (temps), an ${ }^{-1}$ & 0,0019 & 0,0017 \\
\hline Erreur standard résiduelle (23 ddl) & & 0,102 \\
\hline
\end{tabular}

$$
R=0,19+0,0020 . t
$$

L'ordonnée à l'origine $(0,19)$ est assortie d'une erreur standard de 0,035 et la pente $\left(0,0020 \mathrm{an}^{-1}\right)$ d'une erreur standard de $0,0013 \mathrm{an}^{-1}$. L'erreur standard résiduelle est de 0,10 , avec 25 degrés de liberté. À long terme (20 ans), la proportion du carbone nouveau est répartie en $77 \% \pm 4 \%$ en surface et $23 \% \pm 4 \%$ (équation 3). En d'autres termes, la quantité de carbone d'âge inférieur à 20 ans de la couche $30-100 \mathrm{~cm}$ est égale à $0,30 \pm 0,07$ fois celle de la couche $0-30 \mathrm{~cm}$.

\subsection{Distribution verticale des apports de carbone aux matières organiques du sol}

On peut, à partir de cet ensemble de sites, faire des estimations de la répartition verticale des flux d'apports de carbone aux matières organiques. Ceci ne peut être fait individuellement sur chaque site, car chacun a une durée d'observation différente de celle des autres, mais cela peut être fait pour l'ensemble, à partir de la cinétique moyenne d'évolution du rapport entre le carbone nouveau dans chaque couche et le carbone nouveau dans l'ensemble du profil. En effet, la répartition verticale du carbone nouveau est dépendante du temps dans le jeu de données de l'ensemble des sites (Équation 3). Ceci peut être dû à deux phénomènes indépendants :

- le transport progressif de carbone vers la profondeur par bioturbation, lixiviation ou lessivage,

- la biodégradation in situ du C nouveau, plus lente en profondeur qu'en surface.

La dispersion dans le jeu de données ne permet pas de distinguer les deux processus. Cependant, l'estimation globale de la distribution verticale du flux d'apport de carbone aux matières organiques peut être tirée de l'équation 1 . Nous postulons que la répartition du carbone nouveau au temps $t=0$ est une estimation de la distribution verticale du flux d'apport de carbone de la végétation aux matières organiques du sol.

Dans l'ensemble maïs + Miscanthus, ces apports sont répartis en $81 \%$ dans la couche $0-30 \mathrm{~cm}$ et $19 \%$ dans la couche $30-100 \mathrm{~cm}$, avec une erreur standard de $\pm 4 \%$.

Parlemêmecalculderégression, nous pouvons représenter la répartition de ces apports par couche de $10 \mathrm{~cm}$ (Figure 4). Les couches inférieures plus profondes que $40 \mathrm{~cm}$ ont été regroupées par 
Apport de carbone aux $\mathrm{MO}$ du sol (\% de l'apport à 0-100 cm)

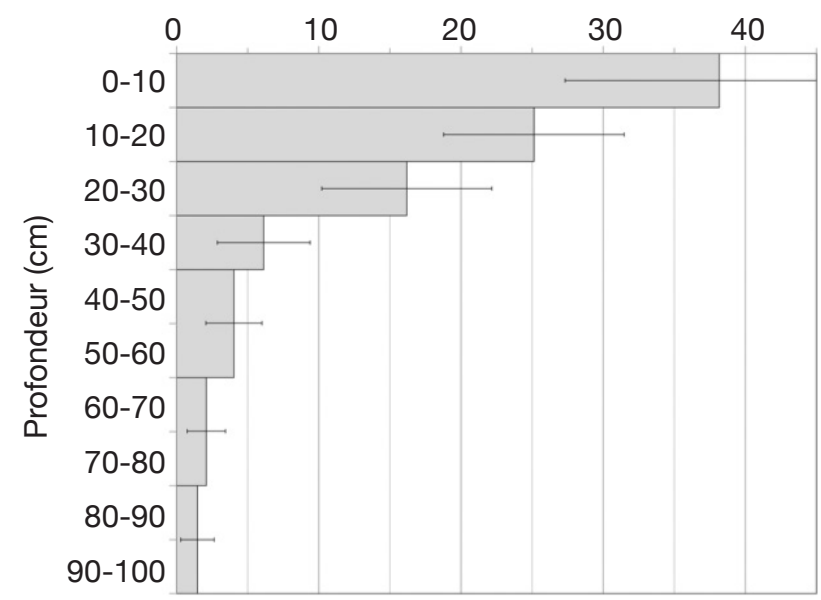

Figure 4. Distribution verticale moyenne du flux d'apport de carbone aux matières organiques des sols cultivés en maïs et Miscanthus - Depth distribution of the input of carbon to organic matter build-up in maize and Miscanthus cropped soils.

Moyennes et écarts-types de 27 à 19 sites, selon la couche considérée - Mean and standard deviation of 27 to 19 sites, depending on the layer.

ensembles de $20 \mathrm{~cm}$ de profondeur dans la présentation pour limiter la dispersion statistique. À l'intérieur de la couche $0-30 \mathrm{~cm}$, la distribution des apports n'est pas uniforme, contrairement à la répartition du carbone total du sol (Figure 2), car la population analysée comprend plusieurs sites non labourés depuis la mise en place de la monoculture: cultures pérennes et conduites en travail du sol réduit.

\section{DISCUSSION}

Il s'agit à notre connaissance d'une première synthèse des mesures de renouvellement du carbone profond sur un tel nombre de sites. Notre étude ne s'appuie que sur quelques cultures et ne peut être extrapolée directement à tous les sites cultivés. Outre les variations d'allocation de carbone souterrain et de développement des systèmes radiculaires, il est attendu que la proportion relative de carbone apporté en profondeur soit d'abord dépendante du régime de restitution des parties aériennes en surface. Dans notre jeu de données, les restitutions sont celles pratiquées le plus classiquement, c'est-à-dire restitution des tiges et feuilles de maïs, restitutions obligatoires à la coupe des Miscanthus. Concernant la canne à sucre, les pratiques ont pu être différentes, avec du brûlage des feuilles fréquemment pratiqué par le passé. Concernant l'ensemble maïs et Miscanthus, nous n'avons pas d'indication que l'allocation souterraine soit significativement différente de celle des céréales, ni même des poacées cultivées en fourrage. En revanche, les légumineuses sont connues pour leur enracinement profond et leur contribution significative au carbone profond a été établie (Guan et al., 2015).

Dans un objectif de modélisation du bilan organique des sols, on pourrait ajouter au bilan du $\mathrm{C}$ superficiel celui du C profond, comme le proposent Jenkinson et al. (2008). Le modèle AMG (Andriulo et al., 1999 ; Bouthier et al., 2014) est l'un des modèles les plus simples du renouvellement du carbone utilisé en agriculture. Le carbone est réparti en un compartiment actif recevant une proportion $k l$ des apports annuels $m$ et une constante de minéralisation $k 2$. À ce carbone actif s'ajoute un compartiment $C_{S}$ de $C$ stable à l'échelle du siècle. On peut ajouter un compartiment de $\mathrm{C}$ actif profond, mais même dans ce cadre très simplificateur, il peut y avoir plusieurs stratégies: représenter ou non un flux de transfert du carbone actif de la surface vers la profondeur, considérer les vitesses de biodégradations $k 2$ comme égales ou non en surface et en profondeur, estimer a priori le $\mathrm{C}$ stable profond, etc. Nous proposons pragmatiquement la représentation la plus simple : vitesses de biodégradation identiques et transport non représenté explicitement. Sur la base des données à moyen terme (20 ans) du Miscanthus et du maïs, cette proposition revient :

- à considérer un compartiment de carbone actif $30-100 \mathrm{~cm}$ égal à $0,30 \quad( \pm 0,07,1 \sigma)$ fois le compartiment actif de la couche $0-30 \mathrm{~cm}$, le reste étant du C stable,

- à ajouter, dans les systèmes avec restitution des résidus de récolte dits « facultatifs », au flux d'apport à la couche $0-30$, un apport à la couche $30-100 \mathrm{~cm}$ égal aussi à 0,30 fois celui alloué à la couche $0-30 \mathrm{~cm}$, - d'appliquer en profondeur les mêmes paramètres $(k l$, $k 2$ ) de la dynamique du compartiment dit « actif » du modèle qu'en surface.

Le transport vertical de carbone est ici intégré implicitement sur 20 ans dans l'ensemble $k 1 m, k 2$. La proportion allouée en profondeur est estimée dans les systèmes avec restitution des parties aériennes dites «facultatives» (tiges et feuilles ou feuilles sénescentes). Il s'agit bien sûr d'une approximation simplificatrice dont on sait qu'elle est probablement biaisée, en raison des vitesses de biodégradation plus lentes en profondeur, du transport vertical de carbone qui peut être très variable et des effets de priming (Fontaine et al., 2007), que l'équation linéaire de minéralisation d'ordre 1 ignore. Un effort de recherche important doit être mené pour paramétrer ces processus.

La variance indiquée ci-dessus montre une très grande incertitude dans l'estimation et donc un risque d'erreur à $95 \%$ sur une estimation isolée proche 
de la moitié du flux lui-même. La variabilité réelle (disparité entre sites) est certainement plus faible que la variabilité observée à partir de notre étude. En effet, une part de la variance vient de la méthode ellemême, notamment de la limite de sensibilité du traçage isotopique. D'autre part, une difficulté récurrente dans l'analyse des profils de sols publiés concerne l'information à la limite inférieure des profils: la plupart des auteurs n'indiquant pas l'épaisseur du sol étudié jusqu'à un horizon de roche mère, si bien qu'il y a un risque de confusion entre sol non échantillonné et absence de terre (concentration en carbone nulle) dans les publications. Nous recommandons de spécifier systématiquement l'épaisseur naturelle du sol et, si possible, d'apporter une description pédologique même sommaire des horizons profonds. Il est certain que les modèles globaux, comme régionaux, de la dynamique du carbone devront bénéficier d'informations détaillées, à la fois sur la profondeur des sols, les caractéristiques pédologiques et les stocks de carbone, comme le propose l'initiative mondiale GlobalSoilMap (Arrouays et al., 2015).

Les valeurs de proportion du carbone incorporé dans les différentes couches que nous rapportons doivent être interprétées en prenant en compte les différents mécanismes contribuant à l'apport de carbone en profondeur. En tout premier lieu et de façon triviale, en cas de labour plus profond que $30 \mathrm{~cm}$, le bilan de carbone doit a minima prendre en compte l'ensemble de la couche homogénéisée pour les bilans de C. Les autres apports sont le transport naturel par bioturbation ou lixiviation et les apports racinaires, les deux pouvant agir en interaction, via le comblement des pores racinaires ou leur colonisation par la faune du sol. Les proportions de carbone alloué aux matières organiques profondes observées ici sont plus importantes que les estimations de biomasse souterraines habituellement rapportées, confirmant l'observation générale qu'à biomasse équivalente, les racines contribuent plus aux matières organiques des sols que les tiges et les feuilles (Balesdent et al., 1996 ; Rasse et al., 2005). Les causes évoquées sont nombreuses, en particulier la contribution des exsudats et rhizodépôts, et la préservation plus importante du carbone issu des tissus racinaires, des composés solubles ou des métabolites microbiens qui en sont issus, en interaction possible avec la sorption et la protection par les phases minérales.

\section{CONCLUSIONS}

Les matières organiques situées en dessous de la couche arable contiennent une partie non négligeable du carbone actif des sols. Nous concluons que ces matières organiques profondes ne doivent être négligées ni dans les bilans de carbone, ni dans l'estimation de la fourniture d'azote par minéralisation des matières organiques. Les rapports $\mathrm{C} / \mathrm{N}$, systématiquement plus petits en profondeur qu'en surface dans les sols, indiquent que les matières organiques profondes sont très azotées et que leur renouvellement dans les couches profondes peut libérer de l'azote minéral dans des proportions proches de celles des flux de carbone, et devrait donc également être pris en compte dans les bilans d'azote.

Dans un objectif de comptabilité du carbone en agriculture, pour le bilan carbone ou pour la gestion de la culture, la prise en compte des variations du C profond est donc nécessaire. Cependant, l'estimation de variations temporelles par mesure directe au champ fait face à des difficultés énormes, largement supérieures à celles rencontrées pour le carbone superficiel, en raison notamment de la variabilité spatiale intra-parcellaire généralement forte, de la lenteur des changements et de l'imprécision sur la mesure des concentrations faibles en carbone (quelques $\mathrm{mg} \cdot \mathrm{g}^{-1}$ ). Les estimations des évolutions temporelles du stock de carbone profond des sols et de leurs facteurs de variation devront donc s'appuyer sur les essais agronomiques de longue durée particulièrement bien contrôlés ou sur la modélisation calée sur des sites fortement instrumentés. Elles devraient être systématiquement accompagnées de descriptions pédologiques pour faciliter leur interprétation. Favoriser les apports de carbone par les systèmes racinaires pour augmenter le stockage de carbone en profondeur est une technique qui mérite attention. Il pourrait s'agir de choix d'espèces ou de génotypes intraspécifiques, prenant en compte également l'interaction entre les génotypes et l'environnement tellurique et pédoclimatique. Il faudra cependant estimer de façon concomitante les conséquences potentielles de l'effet de surminéralisation (priming effect) des matières organiques anciennes du fait de l'apport aux micro-organismes du sol d'exsudats racinaires riches en énergie.

\section{Remerciements}

Cette étude a été partiellement financée par le projet ANR «Dedycas» (Gestion Sobre des Ressources 14-CE010004). Nous remercions Christine Marol, Sylvie Milin, Patrick Signoret et Samuel Legros pour leur contribution aux analyses isotopiques additionnelles et plusieurs scientifiques qui nous ont élégamment fourni les données numériques de leurs données publiées.

\section{Bibliographie}

Ahlström A., Schurgers G., Arneth A. \& Smith B., 2012. Robustness and uncertainty in terrestrial ecosystem carbon response to CMIP5 climate change projections. Environ. Res. Lett., 7, 044008. 
Alexander E.B., 1980. Bulk densities of California soils in relation to other soil properties. Soil Sci.Soc. Am. J., 44, 689-692.

Andriulo A., Mary B. \& Guérif J., 1999. Modelling soil carbon dynamics with various cropping sequences on the rolling pampas. Agron. EDP Sci., 19(5), 365-377.

Arrouays D. et al., 2015. GlobalSoilMap: towards a fineresolution global grid of soil properties. Adv. Agron., 125, 93-134.

Balesdent J. \& Balabane M., 1996. Major contribution of roots to soil carbon storage inferred from maize cultivated soils. Soil Biol. Biochem., 28, 1261-1263.

Balesdent J. \& Mariotti A., 1996. Measurement of soil organic matter turnover using ${ }^{13} \mathrm{C}$ natural abundances. In: Boutton T.W. \& Yamasaki S.I., eds. Mass spectrometry of soils. New York, USA: Marcel Dekker Inc., 83-111.

Balesdent J. et al., 2011. Contribution de la rhizodéposition aux matières organiques du sol, quelques implications pour la modélisation de la dynamique du carbone. Étude Gestion Sols, 18(3), 201-216.

Bouthier A. et al., 2014. Adaptation et mise en oeuvre du modèle de calcul de bilan humique à long terme AMG dans une large gamme de systèmes de grandes cultures et de polyculture-élevage. Innovations Agron., 34, 125 139.

Carvalhais N. et al., 2014. Global covariation of carbon turnover times with climate in terrestrial ecosystems. Nature, 514, 213-217.

Cerri C. et al., 1985. Application du traçage isotopique naturel en ${ }^{13} \mathrm{C}$ à l'étude de la dynamique de la matière organique dans les sols. C. R. Acad. Sci., Ser. II, 9, 423428.

Ciais P.et al., 2013. Carbon and other biogeochemical cycles. In: Stocker T.F. et al., eds. Climate change 2013: the physical science basis. Contribution of Working Group I to the Fifth Assessment Report of the Intergovernmental Panel on Climate Change. Cambridge, UK: Cambridge University Press, 465-570.

Coleman K. \& Jenkinson D.S., 1995. RothC-26 3. A model for the turnover of carbon in soil: model description and users guide. Harpenden, UK: Rothamsted Research.

Elzein A. \& Balesdent J., 1995. Mechanistic simulation of vertical-distribution of carbon concentrations and residence times in soils. Soil Sci. Soc. Am. J., 59, 13281335.

Fontaine S. et al., 2007. Stability of organic carbon in deep soil layers controlled by fresh carbon supply. Nature, 450, 277-280.
Guan X.-K. et al., 2016. Soil carbon sequestration by three perennial legume pastures is greater in deeper soil layers than in the surface soil. Biogeosciences, 3, 527-536.

Guenet B. et al., 2013. The relative importance of decomposition and transport mechanisms in accounting for soil organic carbon profiles. Biogeosciences, 10, 2379-2392.

Hansson P.J., Edwards N.T., Garten C.T. \& Andrews J.A., 2000. Separating root and soil microbial contributions to soil respiration: a review of methods and observations. Biogeochemistry, 48, 115-146.

Harper R.J. \& Tibbett M., 2013. The hidden organic carbon in deep mineral soils. Plant Soil, 368, 641-648.

Hiederer R. \& Köchy M., 2011. Global soil organic carbon estimates and the harmonized world soil database. EUR 25225 EN. Luxembourg: Publications Office of the European Union.

IUSS Working Group WRB, 2014. World Reference Base for Soil Resources 2014. International soil classification system for naming soils and creating legends for soil maps. World Soil Resources Reports No. 106. Roma: FAO.

Jenkinson D.S. \& Coleman K., 2008. The turnover of organic carbon in subsoils. Part 2. Modelling carbon turnover. Eur. J. Soil Sci., 59, 400-413.

Jobbagy E.G.\& Jackson R.B., 2000. The vertical distribution of soil organic carbon and its relation to climate and vegetation. Ecol. Appl., 10, 423-436.

Koven C.D. et al., 2011. Permafrost carbon-climate feedbacks accelerate global warming. Proc. Natl. Acad. Sci. U.S.A., 108, 14769-14774.

Mathieu J., Hatté C., Parent E. \& Balesdent J., 2015. Deep soil carbon dynamics are driven more by soil type than by climate: a worldwide meta-analysis of radiocarbon profiles. Global Change Biol., 21, 4278-4292.

Mulder V.L. et al., 2016. National versus global modelling the $3 \mathrm{D}$ distribution of soil organic carbon in mainland France. Geoderma, 26, 16-34.

Rasse D.P., Rumpel C. \& Dignac M.F., 2005. Is soil carbon mostly root carbon? Mechanisms for a specific stabilisation. Plant Soil, 269, 341-356.

Rumpel C. \& Kögel-Knabner I., 2011. Deep soil organic matter-a key but poorly understood component of terrestrial C cycle. Plant Soil, 338,143-158.

Salomé C. et al., 2010. Carbon dynamics in topsoil and in subsoil may be controlled by different regulatory mechanisms. Global Change Biol., 16, 416-426.

(28 réf.) 


\section{Annexe 1. Liste des études analysées}

Balesdent et al., 1990. Effect of tillage on soil organic carbon mineralization estimated from ${ }^{13} \mathrm{C}$ abundance in maize fields. J. Soil Sci., 41, 587-596.

Bashkin et al., 1998. Changes in soil carbon following afforestation in Hawai. Ecology, 79, 828-833.

Basile-Doelsch I. Unpublished data

Basile-Doelsch et al., 2009. Effect of landuse on organic matter stabilized in organomineral complexes: a study combining density fractionation, mineralogy and $\delta^{13} \mathrm{C}$. Geoderma, 151, 77-86.

Collins et al., 1999. Soil carbon dynamics in corn-based agroecosystems: Results from carbon-13 natural abundance. Soil Sc. Soc. Am. J., 63, 584-591.

Dalal et al., 2005. Total soil organic matter and its labile pools following mulga (Acacia aneura) clearing for pasture development and cropping 1. total and labile carbon. Austral. J Soil Res., 43, 13-20.

Deng et al., 2009. Temporal dynamics of iron-rich, tropical soil organic carbon pools after land-use change from forest to sugarcane. Soils Sediments, 9,112-120.

Felden et al., 2012. Accumulation of Miscanthus-derived carbon in soils in relation to soil depth and duration of land use under commercial farming conditions. J. Plant Nutr. Soil Sci., 175, 661-670.

Flessa et al., 2000. The origin of soil organic C, dissolved organic C and respiration in a long-term maize experiment in Halle, Germany, determined by ${ }^{13} \mathrm{C}$ natural abundance. Plant Nutr. Soil Sci., 163, 157-163.

Gregorich et al., 1995. Turnover of soil organic matter and storage of corn residue carbon estimated from natural ${ }^{13} \mathrm{C}$ abundance. Can. J. Soil Sci., 75, 161-167.

Gregorich et al., 2001. Changes in soil carbon under long-term maize in monoculture and legume-based rotation. Can J. Soil Sci., 81, 21-31.

Hansen et al., 2004. Carbon sequestration in soil beneath long-term Miscanthus plantations as determined by ${ }^{13} \mathrm{C}$ abundance. Biomass Bioenergy, 26, 97-105.

Jantalia et al., 2007. Tillage effect on C stocks of a clayey Oxisol under a soybean-based crop rotation in the Brazilian Cerrado region. Soil Tillage Res., 95, 97-109.

Legros S., 2005. Influence de la phase minérale sur la séquestration du carbone dans les sols d'une climatoséquence de l'Ile de La Réunion. Master Géosystèmes: Universté Paul Cézanne (France).

Lemma et al., 2006. Soil carbon sequestration under different exotic tree species in the southwestern highlands of Ethiopia. Geoderma, 136, 886-898.

Osher et al., 2003. Effect of land use change on soil carbon in Hawaii. Biogeochemistry, 65, 213-232.

Paul A. \& Balesdent J. Unpublished data

Pinheiro et al., 2010. Impact of pre-harvest burning versus trash conservation on soil carbon and nitrogen stocks on a sugarcane plantation in the Brazilian Atlantic forest region. Plant Soil, 333,71-80.

Rasse et al., 2006. Carbon turnover kinetics with depth in a French loamy soil. Soil Sci. Soc. Am. J., 70, 2097-2105.

Romkens et al., 1999. Soil organic matter dynamics after the conversion of arable land to pasture. Biol. Fertil. Soils, 28, 277-284.

Schneckenberger et al., 2007. Carbon sequestration under Miscanthus in sandy and loamy soils estimated by natural ${ }^{13} \mathrm{C}$ abundance. J. Plant Nutr. Soil Sci., 170, 538-542.

Vitorello et al., 1989. Organic matter and natural carbon-13 distribution in forested and cultivated oxisols. Soil Sci. Soc. Am. $J ., 53,773-778$.

Yoneyama et al., 2001. $\delta^{13} \mathrm{C}$ values of organic carbon in cropland and forest soils in Japan. Soil Sci. Plant Nutr., 47,17-26. 